\title{
Experimental Glomerulonephritis in the Isolated Perfused Rat Kidney
}

\author{
William G. Couser, Donald R. Steinmuller, Magda M. Stilmant, \\ David J. Salant, and Leah M. Lowenstein, Evans Memorial Department \\ of Clinical Research and the Departments of Medicine and Pathology, Boston \\ University Medical Center, Boston, Massachusetts 02118
}

\begin{abstract}
A B S T RA C T The development of immune deposits on the subepithelial surface of the glomerular capillary wall was studied in isolated rat kidneys perfused at controlled perfusion pressure, $\mathrm{pH}$, temperature, and flow rates with recirculating oxygenated perfusate containing bovine serum albumin (BSA) in buffer and sheep antibody to rat proximal tubular epithelial cell brush border antigen (FxlA). Control kidneys were perfused with equal concentrations of non-antibody immunoglobulin (Ig)G. Renal function was monitored by measuring inulin clearance, sodium reabsorption, and urine flow as well as BSA excretion and fractional clearance. Perfused kidneys were studied by light, immunofluorescence, and electron microscopy. All kidneys perfused with anti-FxlA developed diffuse, finely granular deposits of IgG along the glomerular capillary wall by immunofluorescence. Electron microscopy revealed these deposits to be localized exclusively in the subepithelial space and slit pores. Similar deposits were produced in a nonrecirculating perfusion system, thereby excluding the formation of immune complexes in the perfusate caused by renal release of tubular antigen. Control kidneys perfused with nonantibody IgG did not develop glomerular immune deposits. Renal function and BSA excretion were the same in experimental and control kidneys. Glomerular deposits in antibody perfused kidneys were indistinguishable from deposits in rats injected with anti-FxlA or immunized with FxlA to produce autologous immune complex nephropathy. These studies demonstrate that subepithelial immune deposits can be produced in the
\end{abstract}

Portions of this work were presented at the 10th Annual Meetings of the American Society of Nephrology, Washington, D. C., 22 November 1977, and at the 7th International Congress of Nephrology, Montreal, Canada, 19 June 1978 and have been published in abstract form in 1977. Kidney Int. 12: 519.

Dr. Couser is the recipient of a National Institutes of Health Research Career Development Award (AM-00102).

Received for publication 30 May 1978 and in revised form 10 August 1978. isolated rat kidney by perfusion with specific antibody to FxlA in the absence of circulating immune complexes. In this model deposits result from in situ complex formation rather than circulating immune complex deposition.

\section{INTRODUCTION}

The role of tissue deposition of immune complexes $(\mathrm{IC})^{1}$ formed in the circulation in the pathogenesis of a variety of clinical and experimental diseases is well documented (1). Granular deposits of immunoglobulin (Ig) and $\beta$ lc- $\beta$ la, third component of complement (C3) in renal glomeruli are considered immunopathologic hallmarks of glomerulonephritis induced by IC deposition (2). Diffuse, finely granular deposits of IgG and $\mathrm{C} 3$ along the subepithelial surface of the glomerular basement membrane (GBM) are characteristic of membranous nephropathy (MN), the most common cause of idiopathic nephrotic syndrome in adults, and are generally believed to result from glomerular trapping of soluble ICs formed in the circulation $(3,4)$. In rats, immunization with an antigen derived from the luminal brush border of proximal tubular epithelial cells (FxlA) results in a glomerular lesion (Heymann nephritis, autologous immune complex nephropathy, AICN) indistinguishable from $\mathrm{MN}$ in man $(5,6)$. A similar lesion is induced more rapidly by administration of heterologous antibody to FxlA (passive Heymann nephritis, PHN) $(7,8)$. The concept that subepithelial immune deposits in these models of $\mathrm{MN}$ represent ICs derived

\footnotetext{
${ }^{1}$ Abbreviations used in this paper: AICN, autologous immune complex nephropathy, Heymann nephritis; BSA, bovine serum albumin; $\mathrm{C} 3, \boldsymbol{\beta}$ lc- $\beta$ la, third component of complement; EM, electron microscopy, FxlA, proximal tubular epithelial cell brush border antigen; GBM, glomerular basement membrane; GFR, glomerular filtration rate; IC, immune complex; IF, immunofluorescence; MN, membranous nephroppathy; PHN, passive Heymann nephritis, heterologous "immune complex" nephropathy.
} 
from the circulation is supported by observations that tubular antigen and antibody to it are present in glomerular deposits and in the circulation in AICN (9-11), and that ICs that contain tubular antigen circulate in AICN rats coincident with the development of glomerular immune deposits (12).

These observations, however, do not document a role for circulating ICs in the formation of subepithelial immune deposits, or exclude alternative explanations for this unique pattern of complex localization. Moreover, several features of the AICN model are at variance with conditions in which circulating ICs localize in the subepithelial space as defined in studies of chronic serum sickness in the rabbit (4). In the latter model subepithelial deposits develop in antigen excess and are usually associated with deposits in the subendothelial and mesangial areas as well (4). In AICN complex deposits develop in marked antibody excess yet are localized exclusively in the subepithelial space $(6,10,13,14)$. Antibody excess complexes are generally of high molecular weight and tend to localize in the mesangium and reticuloendothelial system, although small circulating complexes may form if the antigenic component is oligovalent rather than multivalent. However, another possible explanation for these discrepancies is that subepithelial immune deposits in AICN and MN form in situ. Recent evidence that under appropriate conditions normal rat glomeruli may bind antibody to FxlA in a discontinuous pattern and that direct injection of anti-Fx1A by syringe into a bloodless rat kidney in vivo results in apparent antibody fixation to the glomerular capillary wall support this hypothesis (15). However, the localization of antibody deposits reported in these studies was not characteristic of MN (15).

We have studied the mechanism of formation of subepithelial immune deposits with an isolated perfused rat kidney model because many variables that may influence glomerular transport and localization of macromolecules such as glomerular filtration rate (GFR), perfusion pressure, flow, temperature, $\mathrm{pH}$, and perfusate composition $(16,17)$ can be carefully controlled and monitored in this system. Preliminary data indicating that a glomerular lesion with the ultrastructural and immunofluorescence (IF) features of $\mathrm{MN}$ can be produced in the isolated rat kidney perfused with specific antibody to rat proximal tubular antigen has been presented (18). In the present report our studies of the development of subepithelial immune deposits in the isolated rat kidney are described in detail. They indicate that a glomerular lesion with IF and electron microscopy (EM) features characteristic of MN can be produced experimentally in the absence of circulating ICs.

\section{METHODS}

Preparation of antibody to FxIA. FxlA was prepared from fresh, saline perfused, Sprague-Dawley rat (Charles River
Breeding Laboratories, Inc., Wilmington, Mass.) renal cortices as described by Edgington et al. (19). Male albino rabbits received four immunizations with $20 \mathrm{mg}$ of FxlA emulsified in complete Freund's adjuvant (Difco Laboratories, Detroit, Mich.) given at weekly intervals and were bled 5 days after the last immunization. Adult male sheep were immunized three or four times with $75-100 \mathrm{mg}$ of FxlA in complete Freund's adjuvant over a 2-mo period. Serum was collected 2 wk after the last immunization. All antisera were heat inactivated $\left(56^{\circ} \mathrm{C}, 30 \mathrm{~min}\right)$ and absorbed extensively with pooled, lyophilized whole rat plasma and with rat erythrocytes, leukocytes, and platelets as previously described (20). To eliminate reactivity with $\mathrm{GBM}$ antigens, aliquots of antiserum were further absorbed with purified rat GBM. GBM was prepared from rat glomeruli isolated by differential sieving techniques as described by Spiro (21) and modified by Westberg and Michael (22) and was free of tubular contamination by light microscopy. Isolated glomeruli were washed in distilled water, resuspended in $1.0 \mathrm{M} \mathrm{NaCl}$, and disrupted by sonication on a Branson Sonifier, model W-350 (Branson Sonic Power Company, Danbury, Conn.) at an output setting of six for 3 min in six individual 30-s bursts. After washing three times in $1.0 \mathrm{M} \mathrm{NaCl}$ and distilled water, intact $\mathrm{GBM}$ was lyophilized before use. Antisera were absorbed three times for $1 \mathrm{~h}$ at $37^{\circ} \mathrm{C}$ and overnight at $4^{\circ} \mathrm{C}$ with $0.3 \mathrm{mg}$ of $\mathrm{GBM} / \mathrm{mg} \operatorname{IgG}$. To ensure that this procedure was capable of adequately removing nephrotoxic anti-GBM antibodies if any were present, a potent sheep anti-rat nephrotoxic serum prepared by immunizing sheep with rat glomeruli as described elsewhere (23) was similarly absorbed. Unabsorbed nephrotoxic serum induced $4+$ linear staining of GBM for sheep IgG and a urine protein excretion of $260 \pm 60 \mathrm{mg} /$ day $(n=10)$ in the $24 \mathrm{~h}$ after administration of a standard dose of $0.2 \mathrm{ml}$ to normal rats.

The IgG fraction of antiserum to FxlA was isolated from a $50 \%$ ammonium sulfate precipitate by ion exchange chromatography on DEAE-Sephadex (Pharmacia Fine Chemicals, Piscataway, N. J.) with a $0.0175-\mathrm{M}$ phosphate buffer, $\mathrm{pH} 6.5$. To ensure that the final preparation of antibody used was free of any significant amount of pre-formed ICs, the 7S peak was isolated by gel filtration chromatography on Sephadex G-200 (Pharmacia Fine Chemicals). Further analysis of the composition of the final anti-FxlA antibody and control IgG preparations used were carried out on $1.5 \%$ solutions of IgG in $0.15 \mathrm{M}$ saline by Dr. Karl Schmid (Department of Biochemistry, Boston University School of Medicine) with a Spinco analytical ultracentrifuge, model $\mathrm{E}$, equipped with Schlieren optics and a rotor interval temperature control unit (Beckman Instruments, Inc., Spinco Div., Palo Alto, Calif.) operated at $52,000 \mathrm{rpm}$ at $20^{\circ} \mathrm{C}$. Purified IgG antibody was concentrated to $10 \mathrm{mg} / \mathrm{ml}$ with a stirred ultrafiltration cell and PM30 Diaflow membrane (Amicon Corp. Scientific Sys. Div., Lexington, Mass.) and stored at $-70^{\circ} \mathrm{C}$. Analysis for purity and specificity was carried out by micro-Ouchterlony and immunoelectrophoresis in $1 \%$ agarose $(24,25)$. IgG content was measured by radial immunodiffusion (26). Antibody reactivity was also tested by indirect IF on cryostat sections of normal and AICN rat kidneys (vide infra) (20). Antibody specificity was further documented by repeating studies with antibody absorbed three times with Fx1A, $6 \mathrm{mg} / \mathrm{mg}$ IgG. Normal, unabsorbed sheep or rabbit IgG from nonimmunized animals isolated as described above was used in control studies.

Isolated perfused kidneys. The total number of kidneys perfused in this study and the types of perfusions carried out are listed in Table I. Isolated kidneys were perfused according to the method of Nishiitsutsuji-Uwo et al. (27) and Ross et al. (28). Protein in the perfusate consisted of bovine serum albumin (BSA) (Fraction V, Miles Laboratories, Inc., Miles Research Products, Elkhart, Ind.), previously dialyzed in a $10 \%$ solution (wt/vol) for $48 \mathrm{~h}$ against $30 \mathrm{vol}$ of distilled water and for $12 \mathrm{~h}$ against $30 \mathrm{vol}$ of Krebs-Henseleit bicar- 
TABLE I

Results of IF and EM Studies in Antibody-Perfused and Control Kidneys

\begin{tabular}{|c|c|c|c|c|}
\hline IgG in perfusate* & $\begin{array}{l}\text { Duration } \\
\text { of } \\
\text { perfusion }\end{array}$ & $n \ddagger$ & $\begin{array}{c}\text { GBM } \\
\text { deposits } \\
\text { by IF\$ }\end{array}$ & $\begin{array}{l}\text { Deposits } \\
\text { by EM } \$\end{array}$ \\
\hline & $\min$ & & & \\
\hline \multirow[t]{5}{*}{ Anti-FxlA } & 10 & 2 & 2 & $0 / 1$ \\
\hline & 30 & 2 & 2 & $0 / 1$ \\
\hline & 60 & 3 & 3 & $0 / 2$ \\
\hline & 120 & 8 & 8 & $2 / 3$ \\
\hline & 240 & 1 & 1 & $1 / 1$ \\
\hline \multirow[t]{2}{*}{ Anti-FxlA" } & 30 & 1 & 1 & $0 / 1$ \\
\hline & 60 & 2 & 2 & $1 / 2$ \\
\hline \multirow{2}{*}{$\begin{array}{l}\text { Anti-FxlA (GBM } \\
\text { absorbed) }\end{array}$} & 60 & 1 & 1 & $0 / 1$ \\
\hline & 120 & 2 & 2 & $0 / 1$ \\
\hline \multirow{2}{*}{$\begin{array}{l}\text { Anti-FxlA (FxlA } \\
\text { absorbed) }\end{array}$} & 60 & 1 & 0 & - \\
\hline & 120 & 2 & 0 & $0 / 2$ \\
\hline \multirow[t]{4}{*}{ Normal IgG } & 10 & 2 & 0 & $0 / 1$ \\
\hline & 60 & 2 & 0 & $0 / 1$ \\
\hline & 120 & 3 & 0 & $0 / 2$ \\
\hline & 240 & 2 & 0 & $0 / 1$ \\
\hline \multirow[t]{2}{*}{ Normal IgG" } & 30 & 1 & 0 & - \\
\hline & 60 & 2 & 0 & $0 / 2$ \\
\hline \multirow[t]{6}{*}{ None } & 10 & 3 & 0 & $0 / 1$ \\
\hline & 30 & 3 & 0 & $0 / 1$ \\
\hline & 60 & 4 & 0 & $0 / 2$ \\
\hline & 120 & 4 & 0 & $0 / 2$ \\
\hline & 180 & 2 & 0 & $0 / 1$ \\
\hline & 240 & 3 & 0 & $0 / 2$ \\
\hline
\end{tabular}

* Sheep or rabbit IgG, $30 \mathrm{mg} / 100 \mathrm{ml}$, in $100-150 \mathrm{ml}$ of perfusate.

I $n=$ number of kidneys studied.

$\$$ Number of kidneys positive for granular subepithelial deposits of heterologous IgG.

"Nonrecirculating perfusions.

bonate buffer at $4^{\circ} \mathrm{C}$. The final perfusate consisted of $6.7 \mathrm{~g} /$ $100 \mathrm{ml}$ BSA in Krebs-Henseleit high bicarbonate-buffered saline, $5 \mathrm{mM}$ glucose, and a mixture of amino acids according to de Mello and Maack (29). Aliquots of the perfusate were stored at $-70^{\circ} \mathrm{C}$. Before use the perfusate was filtered through a $0.45-\mu \mathrm{m}$ filter (Millipore Corp., Bedford, Mass.) and circulated through the perfusion system to equilibrate for temperature and oxygen content.

Male Sprague-Dawley rats, 300-450 g (Charles River Breeding Laboratories, Inc.,) were used if the urinary protein excretion was normal $(<5 \mathrm{mg} / 24 \mathrm{~h})$ before study. Rats were anesthetized with an intraperitoneal injection of pentobarbital $(0.5$ $\mathrm{mg} / \mathrm{kg}$ ). The right ureter was cannulated with PE 10 tubing, and $200 \mathrm{U}$ of heparin injected i.v. The right renal artery was cannulated via the superior mesenteric artery. While the kidney was perfused with perfusate (vide infra), the kidney and ureter were dissected free, and the cannula tied in place. The kidney, pedicle, and ureter were then removed en bloc, perfused for $5 \mathrm{~min}$ to remove serum protein, and mounted in the perfusion cabinet.

After perfusion for an equilibration period of $10-15 \mathrm{~min}$, the perfusate volume was adjusted to $100 \mathrm{ml}$. The temperature was maintained at $38^{\circ} \mathrm{C}$ and perfusion pressure was kept at $100 \mathrm{~mm} \mathrm{Hg}$ by adjustment of resistance in a parallel circuit with a screw valve. The $\mathrm{pH}$ was maintained at 7.30-7.40 by automatic titration with $0.1 \mathrm{~N} \mathrm{NaOH}$. Perfusate flow through the isolated kidney measured by strain gauge manometry varied from 30 to $45 \mathrm{ml} / \mathrm{min}$. After the equilibration period $1 \mu \mathrm{Ci}$ of $\left[{ }^{14} \mathrm{C}\right]$ inulin (New England Nuclear, Boston, Mass.) and $30 \mathrm{mg}$ of sheep or rabbit anti-FxlA antibody or control IgG were added to the reservoir. Inulin clearance, sodium reabsorption, water reabsorption, and urine flow were measured at 20 -min intervals. Perfusions lasted up to $4 \mathrm{~h}$ with recirculating perfusate.

To exclude the possibility that ICs were formed in the recirculating perfusate because of release of renal tubular antigens in venous effluent from the isolated kidney, studies were also carried out with nonrecirculating perfusate. These "single pass" perfusions used up to 2 liters of perfusate that contained 10-30 mg/100 ml of antibody or normal IgG and were carried out for a maximum of $50 \mathrm{~min}$.

At selected time intervals tissue obtained by biopsy immediately before the perfusion ended was prepared for light microscopic, IF, and EM studies as outlined below. To systematically compare the findings in the isolated kidney with the glomerular lesion induced by the same antibody in intact animals in our laboratory, rats with AICN and PHN induced as described below were also studied.

The base-line immunopathologic and ultrastructural features of the isolated perfused rat kidney were studied by light, IF, and EM after perfusion of two to four identically prepared kidneys for $10 \mathrm{~min}, 30 \mathrm{~min}, 60 \mathrm{~min}, 2,3$, and $4 \mathrm{~h}$ with no IgG added to the perfusate (Table I).

Induction of AICN and PHN. AICN was induced in 12 150-200 g male Lewis rats (Charles River Breeding Laboratories, Inc.) by a single rear footpad injection of SpragueDawley FxlA in complete Freund's adjuvant (Difco Laboratories) as previously described $(13,20)$. Biopsies of AICN rats 3 and $4 \mathrm{wk}$ after immunization were studied. To define the glomerular lesion induced by anti-Fx1A antibody in the intact animals, PHN was induced with $10 \mathrm{mg}$ of the same sheep or rabbit IgG antibody to FxlA described above and administered i.v. to $20150-200 \mathrm{~g}$ male Sprague-Dawley rats. Renal tissue was studied by IF and EM at intervals from $10 \mathrm{~min}$ to 5 days after antibody injection.

Tissue processing and IF procedures. Tissue obtained by wedge biopsy of isolated perfused kidneys and open renal biopsies in intact animals was processed for examination by light microscopy, IF, and EM as described in detail elsewhere (20). Tissue for light microscopy was fixed in formalin and stained with periodic acid-Schiff, hemotoxylin and eosin, and Jones silver methenamine stains (Jones \& Lamson Products, Waterbury Farrel Div. of Textron Inc., Springfield, Vt.). Colloidal iron and Alcian blue stains carried out at appropriate $\mathrm{pH}$ were done to evaluate glomerular sialoprotein (13).

Direct and indirect IF procedures were performed with reagents, methods, and controls described elsewhere $(20,23)$. All biopsies were stained with monospecific antisera to rat IgG, $\beta$ lc- $\beta$ la (C3), and albumin (13). Antisera to BSA was prepared in rabbits with bovine fraction $\mathrm{V}$ (Miles Laboratories Inc.). The IgG fraction was purified as described above and conjugated with fluorescein isothiocyanate (BioQuest, BBL \& Falcon Products, Div. of Becton, Dickinson \& Co., Cockeysville, Md.) by a dialysis method (30). All sections of perfused kidneys were stained for BSA. The IgG fraction of monospecific antisera to sheep and rabbit IgG were obtained commercially (Cappel Laboratories, Inc., Downingtown, Pa.) and conjugated with fluorescein as described above. The characteristics of rabbit antisera to rat FxlA have been previously reported (20). In addition, aliquots of the sheep antibody to FxlA used for perfusion were also conjugated and used in 

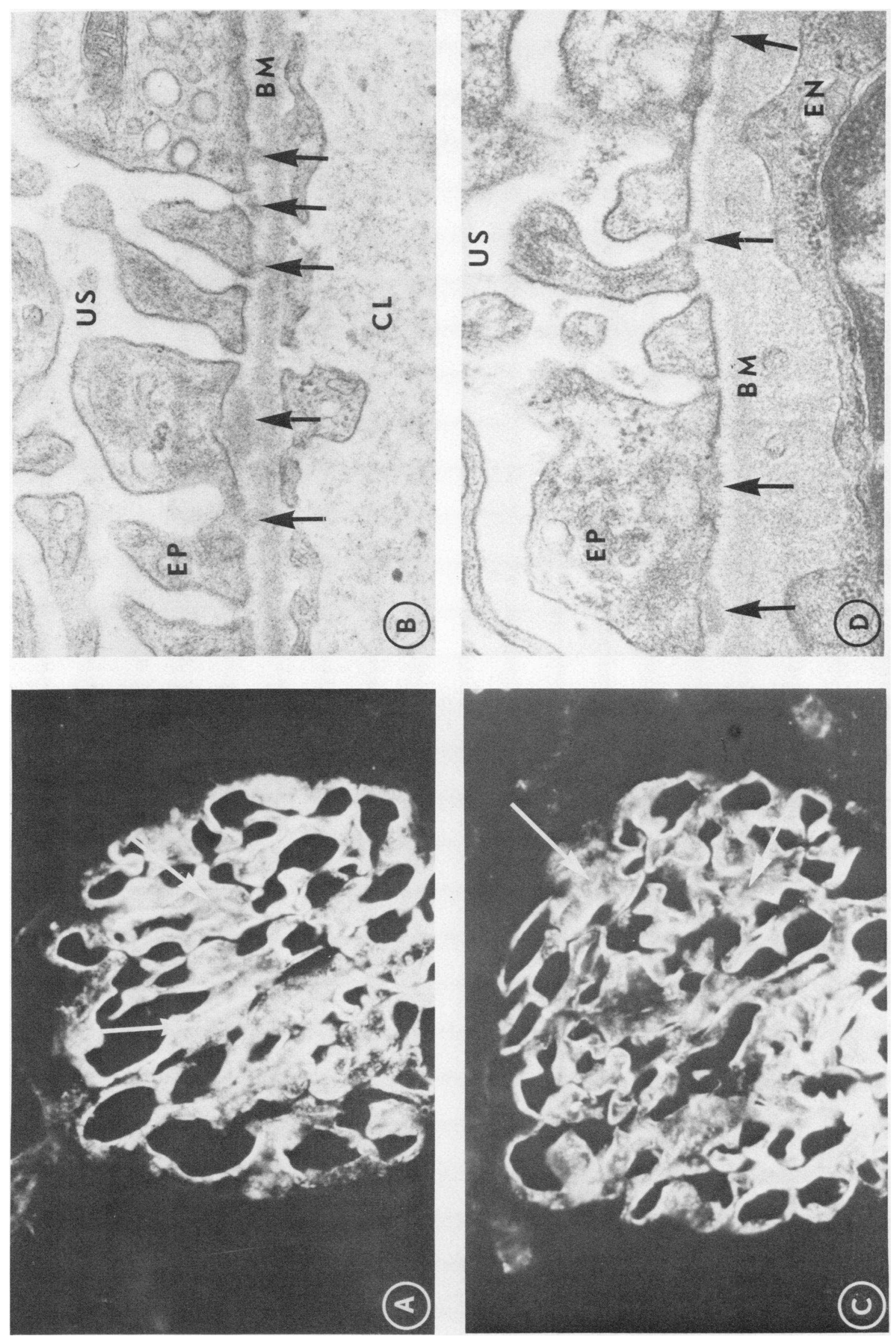
direct IF. The reactivity of these reagents was similar to that of the rabbit antibody used in previous studies (20). IF staining for FxlA was always carried out before and after partial elution of unfixed cryostat sections with $2.5 \mathrm{M}$ potassium thiocyanate at $37^{\circ} \mathrm{C}$ for $120 \mathrm{~min}(9)$.

EM results are based on analysis of 168 sections from 31 perfused kidneys including over 100 sections and electron micrographs from kidneys perfused with normal IgG or antiFxlA (Table I).

Other procedures. Urine protein excretion in intact animals was measured on 24 -h specimens collected in metabolic cages by a sulfosalicyclic method (20). The BSA content of urine produced by the isolated kidney and the concentrations of sheep and rabbit IgG were measured by radial immunodiffusion (26).

\section{RESULTS}

\section{Characteristics of antibody to rat FxlA}

The immunochemical and nephritogenic characteristics of comparable concentrations of sheep and rabbit antibody to $\mathrm{Fx} 1 \mathrm{~A}$ were indistinguishable, and results with both reagents are presented together. When the $7 \mathrm{~S}$ peaks of absorbed antibody and normal IgG were analyzed by micro-Ouchterlony and immunoelectrophoresis they contained only IgG. Ultracentrifugal analysis of the final antibody preparation used revealed that the major component sedimented at $8.5 \mathrm{~S}$. A minor component, which represented only $0.6 \%$ by weight of the antibody preparation and $1.1 \%$ of control IgG, sedimented at $22 \mathrm{~S}$. Anti-Fx $1 \mathrm{~A}$ IgG was not reactive with pooled rat plasma but made two precipitin lines against a $10-\mathrm{mg} / \mathrm{ml}$ suspension of FxlA in saline as described by others $(19,31)$. By indirect IF the antibody reacted exclusively with the luminal brush borders of proximal tubular epithelial cells in normal rat kidney at a concentration of $9.8 \mathrm{mg} / \mathrm{ml}$, was reactive to a dilution of $1 \times 10^{6}$, and did not give detectable staining of normal glomeruli or vessels in vitro. Antibody was also reactive with glomerular deposits in partially eluted sections of AICN kidneys that had 4+ glomerular deposits of IgG $(9,13,20)$. Absorption with Fx1A abolished reactivity with brush borders and AICN glomerular deposits in vitro. Absorption with GBM had no effect on IF reactivity. Control IgG had no demonstrable reactivity with rat plasma, Fx1A, or renal antigens by IF.

\section{AICN rats}

The glomerular lesions in AICN rats at 3 and 4 wk were similar to those described previously $(13,20)$. At 4 wk all rats had 2-3+ deposits of rat IgG and trace-1+ deposits of rat $\mathrm{C} 3$ in a very finely granular pattern in all glomeruli (Fig. 1A). When stained with antisera to FxlA, after partial elution with potassium thiocyanate, Fx1A was also demonstrable in early glomerular deposits $(13,19)$. EM deposits were first detectable 4 wk after immunization and were localized exclusively along the basilar surface of the epithelial cells and in epithelial slit pores $(13,14)$ (Fig. 1B). No mesangial or subendothelial deposits were present. No significant glomerular changes were demonstrable by light microscopy. Staining for glomerular polyanion was not reduced (13). Urine protein excretion in AICN rats at $3-4$ wk averaged $5 \pm 3 \mathrm{mg} /$ day $(n=12)$, and was not significantly different from normal.

\section{Passive Heymann nephritis}

The i.v. administration to intact rats of $10 \mathrm{mg}$ of heterologous IgG antibody to rat FxlA resulted in a diffuse, very finely granular deposition of heterologous IgG in all glomeruli (Fig. 1C). Deposits were apparent in the earliest biopsies at $10 \mathrm{~min}$. These early deposits were so closely spaced as to appear almost linear in some biopsies. Rat C3 was detected by IF in glomerular deposits in a similar pattern. In subsequent biopsies on days 2-5 the amount of heterologous IgG in deposits appeared to increase by IF and the deposits became more clearly granular. These IF deposits of heterologous IgG are compared to the deposits seen in AICN in Fig. 1. No rat IgG or FxlA could be demonstrated in glomerular deposits by IF up to 5 days. Electron dense deposits were first seen by EM 48-72 h after antibody injection (Fig. 1D). Deposits were seen only in the subepithelial space and slit pores and were identical in appearance and localization to early deposits in AICN (Fig. 1). Histologic studies revealed no significant light microscopic changes, no spikes by silver stain, and no alteration in glomerular sialoprotein by histochemical criteria up to 5 days, although urine protein excretion began to increase in antibody injected rats at day $4(28 \pm 12 \mathrm{mg} /$ day, $n=12)$ and reached $52 \pm 10 \mathrm{mg} /$ day on day $5(n=20)$. Except for the absence of detectable rat Fx1A in glomerular immune deposits the glomerular lesions in this model at 3 and 4 days were indistinguishable by light microscopy, IF, and EM from those in AICN rats at about $4 \mathrm{wk}(13,24)$. Control animals injected with normal IgG had no glomerular deposits by IF or EM and did not become proteinuric.

FIGURE 1 Glomeruli of rat with AICN 4 wk after immunization with Fx1A (A and B) with rat with PHN 4 days after injection of $10 \mathrm{mg}$ of sheep anti-rat FxiA (C and D). IF shows very finely granular deposits of rat IgG (A) and sheep IgG (C) on the capillary loops with more homogenous staining in capillary walls cut tangentially (white arrows). EM from the same animals (B and D) shows discreet granular deposits in the subepithelial space and slit pores (dark arrows). (BM: basement membrane, CL: capillary lumen, EN: endothelial cell, EP: epithelial cell, US: urinary space, original magnification: $\mathrm{IF}, \times 630 ; \mathrm{EM} \cong \times 45,000$ ). 


\section{Isolated perfused kidney studies}

Table I lists the types of perfusions carried out in this study and the results of IF and EM studies performed on the perfused kidneys.

Physiologic studies. The values for inulin clearance, sodium reabsorption, and water reabsorption measured at 60 and $120 \mathrm{~min}$ in control and antibody perfused kidneys are shown in Table II. There were no statistically significant differences in these values among the three groups, demonstrating that addition of normal IgG or anti-FxlA to the perfusate did not impair renal function during the experiments.

The values for BSA excretion and fractional clearance in kidneys perfused with normal IgG and antiFxlA are shown in Fig. 2. There was no increase in albumin excretion in kidneys perfused with antibody over that measured in controls.

IF studies. All kidneys perfused with heterologous anti-Fx1A had diffuse, very finely granular deposits of IgG along the capillary walls of all glomeruli (Table I, Fig. 3). Deposits of heterologous IgG were detectable in a granular pattern within $10 \mathrm{~min}$ after the addition of antibody to recirculating perfusions in all the kidneys studied (Fig. 3A, inset). In addition to the finely grannular deposits on capillary walls cut in cross section, a more homogenous deposition of IgG was apparent on areas on basement membrane cut in tangential section (Fig. 3). Careful study of multiple sections disclosed a granular pattern to all staining seen on the capillary wall (Fig. 3). No significant increase in granular staining for IgG was present in the mesangium in antibody-perfused kidneys or controls (Fig. 3). There was an apparent increase in the quantity of immune deposits with duration of perfusion as judged by intensity of IF staining for IgG. The granularity of deposits became clearer with time as described above

TABLE II

Physiologic Parameters of Renal Function Measured at 60 and 120 min in Isolated Kidneys Perfused with No IgG, Non-antibody IgG, and Anti-FxlA in Perfusate*

\begin{tabular}{lcccc}
\hline \multicolumn{1}{c}{ Perfusions } & $n \ddagger$ & $\mathrm{C}_{\mathrm{IN}} \S$ & Urine flow & $\begin{array}{c}\text { Sodium } \\
\text { reabsorption }\end{array}$ \\
\hline 60 min & & $m l / m i n / k i d n e y$ & $\mu l / m i n / k i d n e y$ & $\%$ \\
$\quad$ No IgG & 8 & $0.58 \pm 0.14$ & $74 \pm 4$ & $94 \pm 3$ \\
Normal IgG & 6 & $0.63 \pm 0.13$ & $73 \pm 2$ & $91 \pm 2$ \\
$\quad$ Anti-FxlA & 8 & $0.61 \pm 0.06$ & $69 \pm 4$ & $95 \pm 1$ \\
120 min & & & & \\
No IgG & 8 & $0.38 \pm 0.04$ & $65 \pm 7$ & $92 \pm 3$ \\
Normal IgG & 5 & $0.35 \pm 0.03$ & $54 \pm 3$ & $91 \pm 2$ \\
Anti-FxlA & 8 & $0.44 \pm 0.05$ & $72 \pm 9$ & $93 \pm 2$ \\
\hline
\end{tabular}

* All values are mean \pm SEM.

$\ddagger n=$ number of kidneys studied.

$\S \mathrm{C}_{\mathrm{IN}}$, inulin clearance.
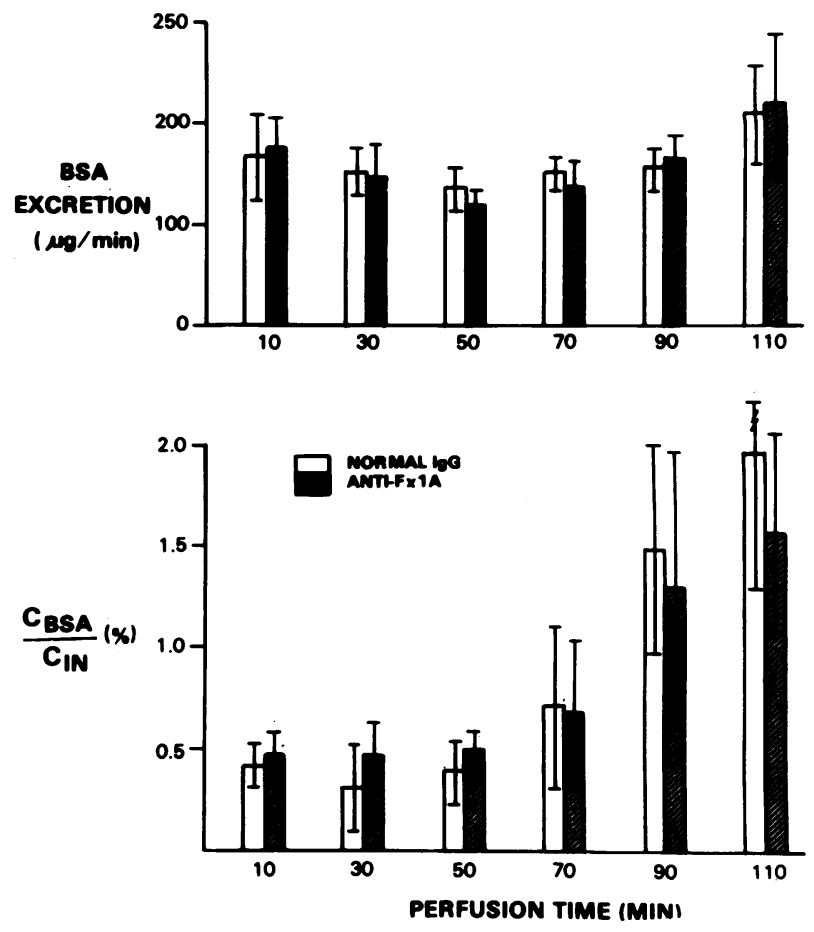

FIGURE 2 BSA excretion and fractional clearances in kidneys perfused with anti-FxlA and normal IgG for up to $2 \mathrm{~h}$. $\mathrm{C}_{\mathrm{BSA}} / \mathrm{C}_{\mathrm{IN}} \%$, bovine serum albumin clearance/inulin clearance, $\times 100$. Mean \pm SEM.

in the intact animal. After $2 \mathrm{~h}$ of recirculating perfusion the amount of glomerular deposits of heterologous IgG by IF was roughly equivalent to that in passively immunized whole animals at 4 days or in AICN rats at 4 wk (Figs. 1, 3). There was no glomerular staining for rat IgG, C3, Fx1A, or for BSA and no binding of heterologous IgG to proximal tubular brush borders was detectable (Fig. 3). In contrast, control kidneys perfused with normal sheep or rabbit IgG showed no deposition of heterologous immunoglobulin in most glomeruli after up to $4 \mathrm{~h}$ of perfusion (Fig. 2B). At 3 and $4 \mathrm{~h}$ rare glomeruli had trace-1+ segmental staining for heterologous IgG in the mesangium. Kidneys perfused without IgG showed no significant glomerular IF deposits. No BSA was detected in reabsorption droplets in tubules.

Recirculating perfusions carried out for 60 and 120 min with sheep anti-FxlA absorbed with GBM showed a pattern of glomerular immune deposits indistinguishable from that described above (Table I). Sheep antirat nephrotoxic serum similarly absorbed with GBM showed complete loss of anti-GBM activity as evidenced by reduction in heterologous phase proteinuria from $260 \pm 40$ to $5 \pm 1 \mathrm{mg} /$ day when doses of $0.2 \mathrm{ml}$ of absorbed serum were administered to groups of 10 normal rats. Linear staining in the intact animal was reduced by GBM absorption from $4+$ to trace- $1+$. Perfusion with 
anti-FxlA after absorption with $\mathrm{Fx} 1 \mathrm{~A}$ resulted in no significant glomerular immune deposits after 60 and 120 min of perfusion (Table I).

Single pass perfusions with sheep anti-FxlA resulted in a pattern of glomerular immune deposits similar to those in the recirculating system but of even greater intensity by IF (Fig. 4A). As in the recirculating system no glomerular localization of heterologous nonantibody IgG could be demonstrated in controls (Fig. 4B). No FxlA was demonstrable in glomeruli of kidneys perfused in this way and no tubular brush border staining was detectable (Fig. 4).

EM studies. Kidneys perfused without IgG for up to $2 \mathrm{~h}$ (14 kidneys) and 2 kidneys perfused for $4 \mathrm{~h}$ showed remarkable preservation of glomerular architecture by EM. Epithelial cell foot processes remained well defined and there were no significant electron dense deposits. No deposits could be detected by EM in kidneys perfused with normal IgG or with anti-FxlA for less than $2 \mathrm{~h}$ although immune deposits were readily demonstrable in antibody perfused kidneys by IF, a finding similar to that reported previously in AICN rats (13). Kidneys perfused for $2 \mathrm{~h}$ or longer with antiFxlA demonstrated multiple, small electron-dense deposits localized exclusively in the subepithelial space, along the basilar portion of epithelial cells, and in slit pores (Fig. 3C) $(13,41)$. Although smaller in size, these deposits in antibody-perfused kidneys were identical in localization and distribution to those seen in AICN rats at $4 \mathrm{wk}$ and in PHN rats 4 days after injection of $10 \mathrm{mg}$ of the same antibody (Figs. 1,3). No mesangial deposits were seen by EM in antibody-perfused kidneys.

Histologic studies. Histologic abnormalities induced by the perfusion procedure per se were assessed by light microscopy in 19 control kidneys studied after $10 \mathrm{~min}$ to $4 \mathrm{~h}$ of recirculating perfusion. Glomeruli had no detectable morphologic alterations and glomerular sialoprotein was normal by histochemical staining in all perfused kidneys. Some kidneys showed focal tubular dilatation and mild interstitial edema as described by others (32). There were no histologic differences between kidneys perfused with no IgG, normal IgG, and anti-FxlA antibody. Silver methenamine stains of antibody perfused kidneys did not show spike formation.

\section{DISCUSSION}

These results clearly establish that the immunopathologic and ultrastructural features of the glomerular lesion in AICN and PHN, which are indistinguishable from those of $\mathrm{MN}$ in man, can be produced experimentally in the rat kidney by perfusion under controlled conditions with antibody to proximal tubular brush border antigens, and hence must have been initiated by in situ complex formation rather than circulating
IC deposition. The specificity of this finding was confirmed by the absence of subepithelial immune deposits in kidneys perfused under similar conditions with equivalent amounts of nonantibody IgG and with antibody absorbed with Fx1A. The possibility that preformed ICs were added to the perfusate with the antibody was excluded by isolation of the $7 \mathrm{~S}$ fraction of the antibody preparations used by gel filtration and by ultracentrifugal analysis of the final antibody preparations. Moreover, the findings in the nonrecirculating system exclude any possibility that soluble ICs formed in the perfusate contributed to the glomerular deposits produced. Thus, the finely granular, subepithelial immune deposits in this model represent the initiation of complex formation by binding of antibody to antigens apparently localized on the epithelial aspects of the glomerular capillary wall. In view of the failure to demonstrate specific antigen in glomeruli or deposits, however, the possibility that nonimmunologic physical-chemical events may contribute to antibody deposition at this site must also be considered. An in situ process of complex formation has previously been demonstrated in basement membranes of thyroid, testis, and renal tubules (33-36). A similar mechanism has also been postulated to result in glomerular deposits in systemic lupus (37).

The demonstration that subepithelial complex deposits can develop in situ in the glomerulus does not document a role for this mechanism in mediating AICN or PHN or exclude a role for the deposition of circulating ICs in the pathogenesis of glomerular injury in these models. However, previous studies in support of a role for circulating IC deposition in experimental MN must now be re-evaluated. These include the following observations. Antibody to tubular antigen disappears from the circulation of normal rats more rapidly than nonantibody IgG (38). Tubular antigen and antibody to it can be identified in the circulation $(10,11,38)$ and in glomerular deposits in AICN rats $(9,10)$. The development of complex deposits in normal kidneys transplanted into rats injected with antibody to tubular brush border antigen 1-3 days earlier has been interpreted as evidence of persistent circulating ICs in the PHN model (31). Tubular antigen and IgG in a granular, presumably IC form, are deposited in the glomerular mesangium of AICN rats made proteinuric with aminonucleoside of puromycin (20). ICs of similar composition have also been demonstrated in the circulation of AICN rats (12). Considered together, all of these immunopathologic observations appear as consistent with the hypothesis that deposits in the subepithelial space in experimental MN represent in situ complex formation as with the interpretation that they represent ICs derived from the circulation. Although there is currently little evidence that the immunopathogenesis of human $\mathrm{MN}$ is similar to that in the 

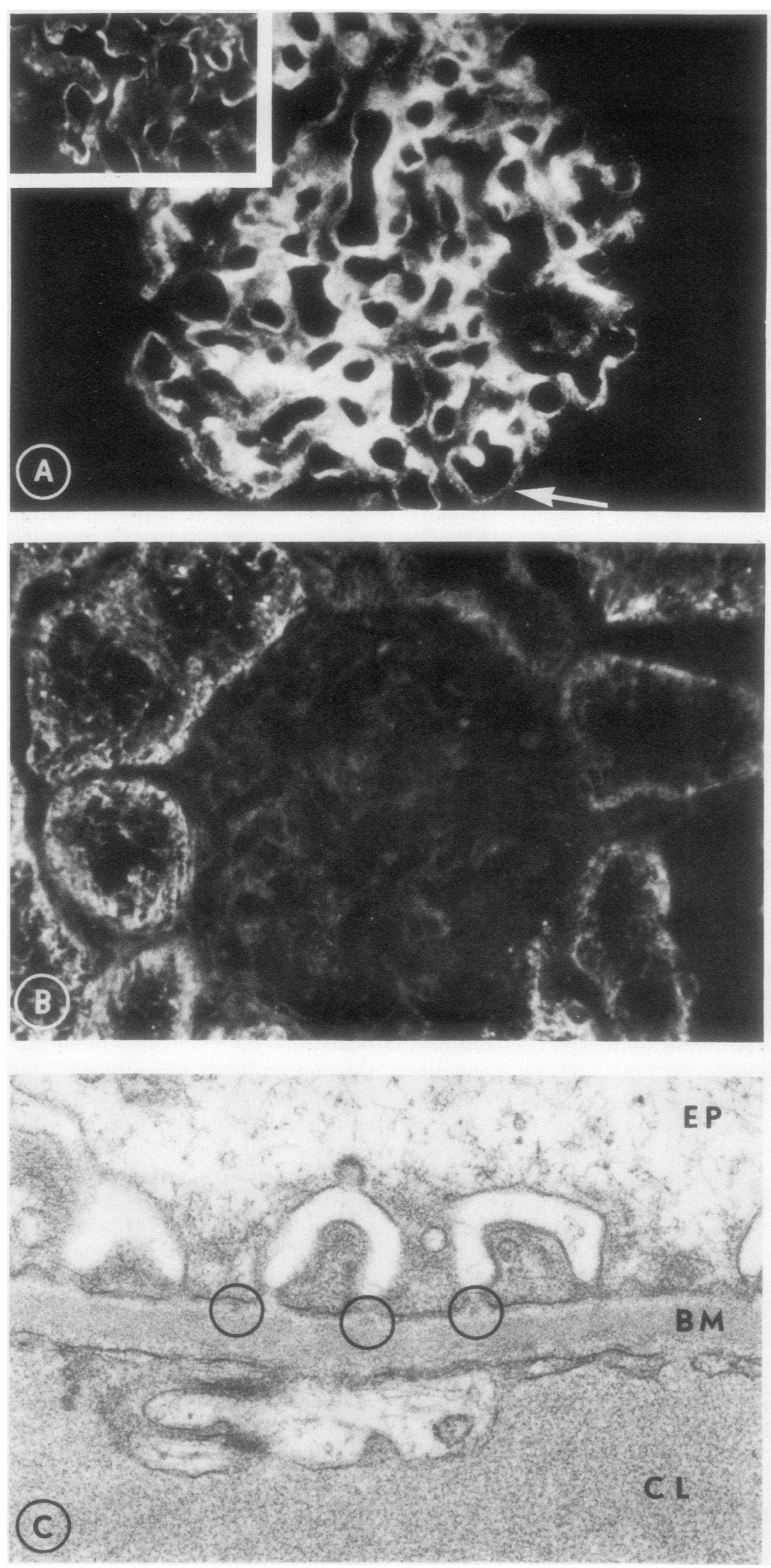
AICN or PHN models, an in situ mechanism of complex formation is consistent with several studies showing circulating ICs to be absent in idiopathic $\mathrm{MN}$ in man $(39-41)$ or present less frequently than in other renal diseases not associated with glomerular immune deposits $(42,43)$.

A role for soluble ICs in the pathogenesis of experimental MN has also been suggested by the observations that IF and EM deposits occur in a subepithelial distribution in some rabbits with chronic serum sickness $(4,44)$. In this model complex localization at an epimembranous site occurs only when low molecular weight ICs are formed in persistent antigen excess (4). Complex deposition is almost always present in subendothelial and mesangial areas as well (4). These findings are in marked contrast to the AICN and PHN models where deposits develop in marked antibody excess $(10,20,31,38)$ but localize only in the subepithelial space $(6,8,13,14)$. Complex deposition is not altered in PHN by varying antibody levels (45), a manipulation which should change IC size and localization if the antigen is multivalent, and antigen excess results in an acute reduction rather than an increase in basement membrane deposits (46). Treatment with antihistamines has also been shown not to alter complex formation in PHN (45). Moreover, numerous attempts to induce IC nephritis by passive infusion of ICs of appropriate size have failed to demonstrate that pre-formed ICs can be filtered from the circulation and localize in the subepithelial space $(15,47-50)$. Stilmant et al. have provided the only tentative evidence that such a phenomenon can occur (51). In their studies, mice immunized with ferritin developed circulating, ferritin-containing ICs, identified by ultrastructural rather than immunochemical criteria, which appeared to cross the GBM to localize in the subepithelial space. However, more deposits were localized in subendothelial and mesangial areas (51). Currently published data thus, do not exclude the possibility that the subepithelial deposits which develop in chronic serum sickness may also represent in situ complex formation which occurs only when the antigenic component of the complex is present in free form and therefore able to penetrate the GBM to serve as a fixed antigen. Larger aggregates resulting from intravascular IC formation probably localize exclusively in subendothelial and mesangial areas or outside the kidney.
Potentially antigenic macromolecules of appropriate molecular size and charge, such as cationic ferritin, lysozyme, and heparin-protamine polyelectrolyte complexes, can localize by nonimmunologic (probably charge related) mechanisms on the epithelial surface and in slit pores of a normal glomerulus (52-55). The distribution of these electron-dense substances resembles that of immune deposits in early AICN or PHN $(8,13,14)$. IgG can apparently penetrate the glomerular capillary wall to the subepithelial space when blood flow is reduced (56). GBM penetration by IgG under conditions of normal flow may resemble that of ferritin if antibody is present in subgroups with sufficiently cationic isoelectric points (52). The selective concentration of the anionic ultrastructural tracer molecules, catalase and ferritin, with isoelectric points of 5.7 and 4.6, respectively, in subepithelial deposits in AICN suggests that these deposits are cationic relative to normal GBM (57). Thus, in situ formation of complexes in a discontinuous pattern on the subepithelial surface of the GBM is consistent with known mechanisms of glomerular transport and distribution of macromolecules.

With regard to the probable glomerular localization of the antigen involved in the AICN and PHN models, our studies are consistent with the hypothesis that it is a constituent of the capillary wall that is immunologically cross reactive with FxlA. Electron dense deposits in our studies were identified in the subepithelial space in a distribution identical to that in early AICN and PHN. Deposits were not seen at other sites within the capillary wall as reported by Van Damme et al. with an immunoperoxidase technique (15). This apparent discrepancy may relate to the larger quantity of antibody deposits in our studies during $2 \mathrm{~h}$ of continuous antibody perfusion. Deposits could be visualized by conventional EM, thus obviating the need to determine antibody localization with multilayer immunoenzymatic techniques with multiple potential artifacts. Antibody binding was not reduced by absorption with GBM prepared by sonic disruption of whole glomeruli. This suggests the antigen may be a constituent of the epithelial cell surface, because GBM prepared by this technique has shown to be relatively free of epithelial cell contamination as judged by phospholipid content $(21,25)$. Although we could not demonstrate binding of anti-FxlA antibody to normal glomeruli in

FIGURE 3 Representative IF and EM pictures from glomeruli of isolated rat kidneys perfused for $2 \mathrm{~h}$ with $30 \mathrm{mg} / 100 \mathrm{ml}$ of anti-FxlA IgG (A and C) or nonantibody IgG (B). A. IF in antibody perfused kidneys shows diffuse, very finely granular deposition of sheep IgG along all capillary walls. In some loops closely spaced deposits along the epithelial surface of the capillary wall are clearly apparent (white arrows) $(\times 630)$. Inset demonstrates finely granular deposits along the epithelial surface of capillary loops in a biopsy obtained only 10 min after initiation of antibody perfusion $(\times 630)$. B. Kidney perfused with normal sheep IgG for $2 \mathrm{~h}$ shows no glomerular deposition of sheep IgG $(\times 630)$. C. EM of the same antibody perfused kidneys shown in A demonstrates multiple small electron dense deposits in the subepithelial space and slit pores (circles). (BM, CL and EP as in Fig. 1. Original magnification $\cong \times 38,000$ ). 

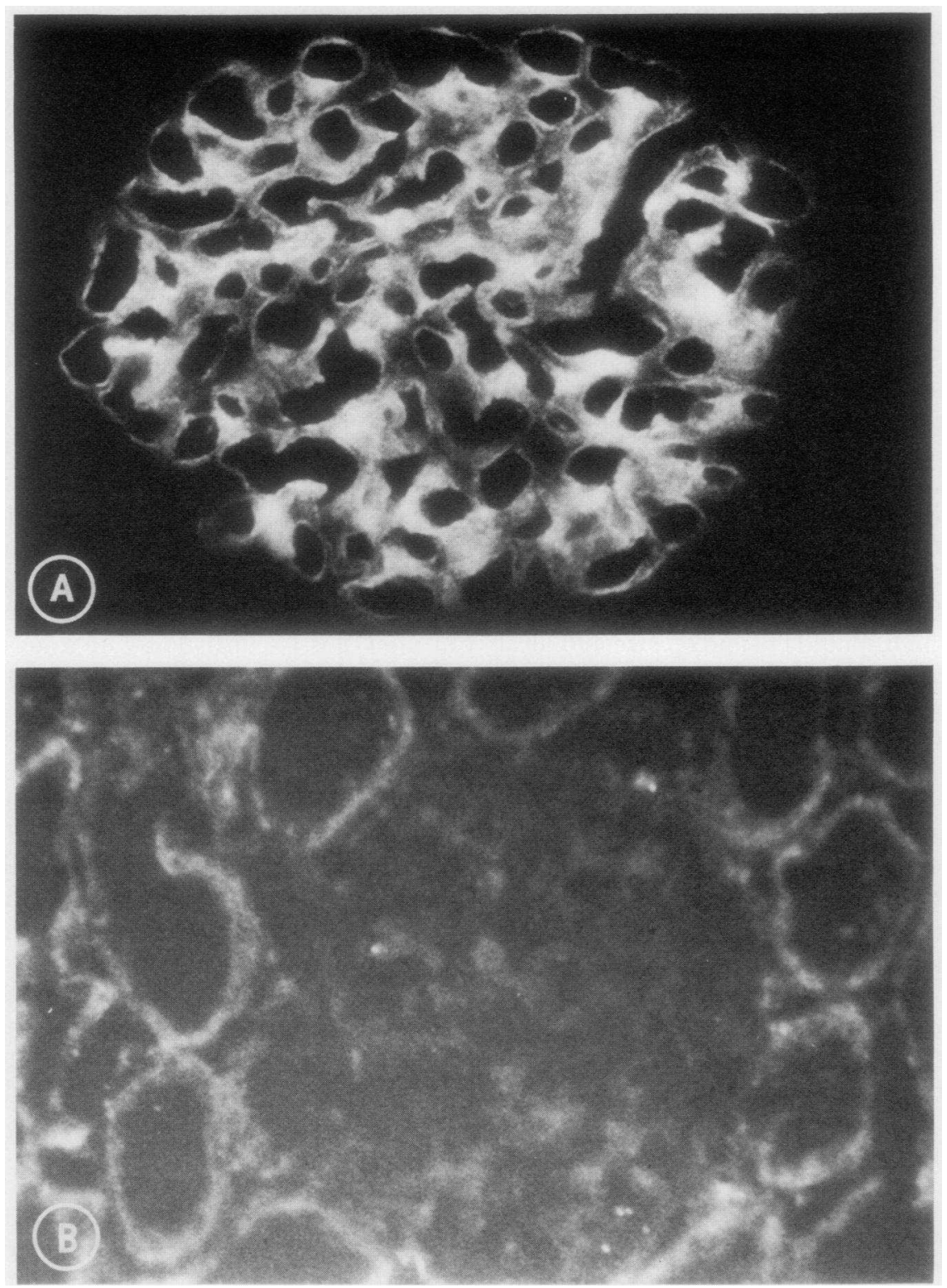

FIGURE 4 IF photographs of glomeruli perfused for $30 \mathrm{~min}$ with non-recirculating perfusate containing anti-Fx1A (A) and normal sheep IgG (B). Antibody perfused kidney shows diffuse finely granular deposits of sheep IgG on the capillary walls (A). Kidney perfused with normal IgG shows no glomerular IgG localization $(B)(\times 630)$.

cryostat sections, others have reported glomerular staining in vitro with similar reagents after pretreatment of glomeruli with neuraminidase and acid buffers (15). Additional support for the presence of an antigen in the rat glomerulus which can induce a membranous lesion is provided by the studies of Shibata et al. who produced a lesion very similar to AICN by immunizing rats with a highly purified fraction of pronase-digested GBM (58).

Our results do not clarify the mechanism by which subepithelial immune deposits increase glomerular permeability in the intact animal. The absence of a measurable increase in protein excretion in kidneys perfused with antibody compared to controls is consistent with observations in the intact animal in which comparable amounts of subepithelial immune deposits do not produce proteinuria $(13,20)$. Some of the protein excreted by the isolated perfused kidney noted by us and others (27) may be nonglomerular in origin (59).

In contrast to other studies (15) the electrolyte and protein content of perfusate, perfusion pressure, temperature, and $\mathrm{pH}$ in the system used in our experiments 
all approximated in vivo conditions. The parameters of kidney structure and function measured were not altered during perfusion with antibody and were similar to those reported by other workers in control kidneys (27-29). GFR of perfused kidneys was about $50 \%$ of that measured in vivo. The low GFR, also found by others, has been attributed to elevated proximal intratubular hydrostatic pressure, uneven distribution of perfusion, mechanical occlusion of glomerular capillaries, and abnormal leakiness of distal nephrons (60). None of these factors appeared to influence the development of glomerular immune deposits in antibody perfused kidneys since GFR was the same in controls. The possibility that ICs formed because of release of tubular brush border antigen into perfusate was excluded in our studies by demonstrating that similar deposits developed in nonrecirculating perfusions when renal venous effluent was discarded. The increase in renal plasma flow by a factor of 6-10 in isolated kidneys over values in vivo may explain why more deposits are present in the isolated kidney at $2 \mathrm{~h}$ than in the antibody injected whole animal $(1,61)$.

Our findings demonstrate that in experimental MN the granular pattern of complex deposition believed to be indicative of nephritis mediated by glomerular trapping of circulating ICs, may instead represent antibody binding to antigens present or previously localized in the glomerular capillary wall. If a similar mechanism is operative in vivo, AICN may be more analogous to experimental auto-immune glomerulonephritis or nephrotoxic nephritis induced by anti-GBM antibody than to the chronic serum sickness model with which it is commonly compared (4). The possibility is supported by other studies of the determinants of subepithelial complex localization in this model $(45,46)$. An in situ mechanism of complex formation in MN could have considerable therapeutic significance since the factors known to influence soluble IC localization are different from those affecting antibody binding to fixed tissue antigens (62).

\section{ACKNOWLEDGMENTS}

The authors are grateful to Christine Darby, Karen Steele, Vita Sliogeris, and Mary Moran for expert technical assistance and to Lisa Medoff for secretarial support in preparation of the manuscript.

Support for this work was provided by research grants AM 17722, AM 17713, AG 00449, and HL 18313, and National Research Training Grant AM 07053 from the U. S. Public Health Service and a grant from the John A. Hartford Foundation.

\section{REFERENCES}

1. Cochrane, C. G., and D. Koffler. 1973. Immune complex disease in experimental animals and man. Adv. Immunol. 16: $185-264$.

2. Wilson, C. B., and F. J. Dixon. 1974. Diagnosis of immunopathologic renal disease. Kidney Int. 5: 389-401.
3. Rosen, S. 1971. Membranous glomerulonephritis: Current status. Hum. Pathol. 2: 209-231.

4. Germuth, F. G., Jr., and E. Rodriquez. 1973. Immunopathology of the renal glomerulus. Little, Brown \& Co., Boston. 25-42.

5. Heymann, W., D. B. Hackel, J. Harwood, S. G. F. Wilson, and J. L. P. Hunter. 1959. Production of nephrotic syndrome in rats by Freunds adjuvants and rat kidney suspensions. Proc. Soc. Exp. Biol. Med. 100: 660-666.

6. Alousi, M. A., R. S. Post, and W. Heymann. 1969. Experimental autoimmune nephrosis in rats. Morphogenesis of the glomerular lesion: Immunohistochemical and electron microscopic studies. Am. J. Pathol. 54: 47-71.

7. Barabas, A. Z., and R. Lannigan. 1974. Induction of an autologous immune-complex glomerulonephritis in the rat by intravenous injection of heterologous anti-rat kidney tubular antibody. I. Production of chronic progressive immune-complex glomerulonephritis. Br. J. Exp. Pathol. 55: 47-55.

8. Feenstra, K., R. v. d. Lee, H. A. Greben, A. Arends, and $\mathrm{Ph}$. J. Hoedemaeker. 1975. Experimental glomerulonephritis in the rat induced by antibodies directed against tubular antigens. I. The natural history: a histologic and immunohistologic study at the light microscopic and ultrastructural level. Lab. Invest. 32: 235-242.

9. Edgington, T. S., R. J. Glassock, and F. J. Dixon. 1967. Autologous immune complex pathogenesis of experimental allergic glomerulonephritis. Science (Wash. D.C.). 155: 1432-1434.

10. Grupe, W. E., and M. H. Kaplan. 1969. Demonstration of an antibody to proximal tubular antigen in the pathogenesis of experimental autoimmune nephrosis in the rat. J. Lab. Clin. Med. 74: 400-409.

11. Naruse, T., T. Fukasawa, N. Hirokawa, S. Oike, and Y. Miyakawa. 1976. The pathogenesis of experimental membranous glomerulonephritis induced with homologous nephritogenic tubular antigen. J. Exp. Med. 144: 13471362.

12. Abrass, C. K., W. A. Border, and R. J. Glassock. 1977. Demonstration of circulating immune complexes (CIC) in autologous immune complex nephritis (AICN) in rats. Kidney Int. 12: 508. (Abstr.)

13. Couser, W. G., M. M. Stilmant, and C. Darby. 1976. Autologous immune complex nephropathy. I. Sequential study of immune complex deposition, ultrastructural changes, proteinuria and alterations in glomerular sialoprotein. Lab. Invest. 34: 23-30.

14. Schneeberger, E. E., and W. E. Grupe. 1976. The ultrastructure of the glomerular slit diaphragm in autologous immune complex nephritis. Lab. Invest. 34: 298-305.

15. Van Damme, B. J. C., G. J. Fleuren, W. W. Bakker, R. L. Vernier, and Ph. J. Hoedemaeker. 1978. Experimental glomerulonephritis in the rat induced by antibodies directed against tubular antigens. V. Fixed glomerular antigens in the pathogenesis of heterologous immune complex glomerulonephritis. Lab. Invest. 38: 502-510.

16. Ryan, G. B., and M. J. Karnovsky. 1976. Distribution of endogenous albumin in the rat glomerulus: Role of hemodynamic factors in glomerular barrier function. Kidney Int. 9: 36-45.

17. Deen, W. M., M. P. Bohrer, C. R. Robertson, and B. M. Brenner. 1977. Determinants of the transglomerular passage of macromolecules. Fed. Proc. 36: 2614-2618.

18. Steinmuller, D. R., S. Belok, M. M. Stilmant, L. M. Lowenstein, and W. G. Couser. 1977. Experimental glomerulonephritis in the isolated perfused rat kidney (IPK). Kidney Int. 12: 519. (Abstr.)

19. Edgington, T. S., R. J. Glassock, and F. J. Dixon. 1968. Antologous immune complex nephritis induced with 
renal tubular antigen. I. Identification and isolation of the pathogenetic antigen. J. Exp. Med. 127: 555-572.

20. Couser, W. G., J. R. Hoyer, M. M. Stilmant, N. B. Jermanovich, and S. Belok. 1978. The effect of aminonucleoside nephrosis on immune complex localization in autologous immune complex nephropathy in rats. J. Clin. Invest. 61: 561-572.

21. Spiro, R. G. 1967. Studies on the renal glomerular basement membrane. Preparation and chemical composition. J. Biol. Chem. 242: 1915-1922.

22. Westberg, N. B., and A. F. Michael. 1970. Human glomerular basement membrane. Preparation and composition. Biochemistry. 9: 3837-3846.

23. Couser, W. G., M. M. Stilmant, and N. B. Jermanovich. 1977. Complement-independent nephrotoxic nephritis in the guinea pig. Kidney Int. 11: 170-180.

24. Ouchterlony, O. 1970. Handbook of Immunodiffusion and Immunoelectrophoresis. Ann Arbor-Humphrey Science Publishers, Inc., London. 21-31.

25. Ouchterlony, O. 1970. Handbook of Immunodiffusion and Immunoelectrophoresis. Ann Arbor-Humphrey Science Publishers, Inc., London. 47-57.

26. Mancini, O., A. O. Carbonara, and J. R. Heremans. 1965. Immunochemical quantitation of antigens by single radial immunodiffusion. Immunochemistry. 2: 235-254.

27. Nishiitsutsuji-Uwo, J. M., B. D. Ross, and H. A. Krebs. 1967. Metabolic activities of the isolated perfused rat kidney. Biochem. J. 103: 852-862.

28. Ross, B. D., F. H. Epstein, and A. Leaf. 1973. Sodium reabsorption in the perfused rat kidney. Am. J. Physiol. 225: $1165-1171$

29. de Mello, G., and T. Maack. 1976. Nephron function of the isolated perfused rat kidney. Am. J. Physiol. 231: 1699-1707.

30. Clark, H. F., and N. C. Shepard. 1963. A dialysis technique for preparing fluorescent antibodies. Virology. 20: $642-644$.

31. Van Es, L. A., A. P. R. Blok, L. Schoenfeld, and R. J. Glassock. 1977. Chronic nephritis induced by antibodies reacting with glomerular-bound immune complexes. Kidney Int. 11: 106-115.

32. Bauman, A. W., T. W. Clarkson, and E. M. Miles. 1963. Functional evaluation of isolated perfused rat kidney. $J$. Appl. Physiol. 18: 1239-1246.

33. Clagett, J. A., C. B. Wilson, and W. O. Weigle. 1974. Interstitial immune complex thyroiditis in mice. The role of autoantibody to thyroglobulin. J. Exp. Med. 140: 14391456.

34. Bigazzi, P. E., L. L. Kosuda, K. C. Nsu, and G. A. Andres. 1976. Immune complex orchitis in vasectomized rabbits. J. Exp. Med. 143: 382-404.

35. Klassen, J., R. T. McCluskey, and F. Milgrom. 1971. Nonglomerular renal disease produced in rabbits by immunization with homologous kidney. Am. J. Pathol. 63: 333358.

36. Hoyer, J. R. 1976. Autoimmune tubulointerstitial nephritis induced in rats by immunization with rat TammHorsfall urinary glycoprotein. Kidney Int. 10: 544. (Abstr.)

37. Izui, S., P. H. Lambert, and P. A. Miescher. 1976. In vitro demonstration of a particular affinity of glomerular basement membrane and collagen for DNA. A possible basis for local formation of DNA-anti-DNA complexes in systemic lupus erythematosus. J. Exp. Med. 144: 428-443.

38. Glassock, R. J., T. S. Edgington, J. I. Watson, and F. J. Dixon. 1968. Autologous immune complex nephritis induced with renal tubular antigen. II. The pathogenetic mechanism. J. Exp. Med. 127: 573-588.

39. Ooi, Y. M., B. S. Ooi, V. E. Pollak. 1977. Relationship of levels of circulating immune complexes to histologic patterns of nephritis: A comparative study of membranous glomerulonephropathy and diffuse proliferative glomerulonephritis. J. Lab. Clin. Med. 90: 891-898.

40. Tung, K. S. T., A. J. Woodroffe, T. D. Ahlin, R. C. Williams, Jr., and C. B. Wilson. 1978. Application of solid phase Clq and Raji cell radioimmune assays for the detection of circulating immune complexes in glomerulonephritis. J. Clin. Invest. 62: 61-72.

41. Zager, R. A., W. G. Couser, B. S. Andrews, W. K. Bolton, and M. A. Pohl. 1978. Membranous nephropathy: A radioimmunologic search for anti-tubular epithelial antibodies and circulating immune complexes. Nephron. In press.

42. Woodroffe, A. J., W. A. Border, A. M. Theofilopoulos, O. Gotze, R. J. Glassock, F. J. Dixon, and C. B. Wilson. 1977. Detection of circulating immune complexes in patients with glomerulonephritis. Kidney Int. 12: 268-278.

43. Border, W., C. Abrass, C. Hall, C. Brown, R. Glassock, and C. Coggins. 1977. Detection of circulating immune complexes (CIC) in adult idiopathic nephrotic syndrome (AINS). Kidney Int. 12: 510. (Abstr.)

44. Dixon, F. J., J. D. Feldman, and J. J. Vazquez. 1971. Experimental glomerulonephritis. The pathogenesis of a laboratory model resembling the spectrum of human glomerulonephritis. J. Exp. Med. 113: 889-919.

45. Belok, S., D. R. Steinmuller, D. J. Salant, C. Darby, M. M. Stilmant, and W. G. Couser. 1977. Determinants of glomerular localization of subepithelial immune deposits. Kidney Int. 12: 510. (Abstr.)

46. Fleuren, G. J., R. v. d. Lee, H. A. Greben, B. J. C. Van Dainme, and $\mathrm{Ph}$. J. Hoedemaeker. 1978. Experimental glomerulonephritis in the rat induced by antibodies directed against tubular antigens. IV. Investigations into the pathogenesis of the model. Lab. Invest. 38: 496-501.

47. Cochrane, C. G. 1971. Mechanisms involved in the deposition of immune complexes in tissues. J. Exp. Med. 134(Pt. 2): $75 \mathrm{~s}-89 \mathrm{~s}$.

48. Okumura, K., Y. Kondo, and T. Tada. 1971. Studies on passive serum sickness. I. The glomerular fine structure of serum sickness nephritis induced by preformed antigenantibody complexes in the mouse. Lab. Invest. 24: 383391.

49. O'Regan, S., M. Smith, and K. N. Drummond. 1976. Immune complex infusion in the rat. Renal functional and morphological changes. Clin. Exp. Immunol. 24: 110115.

50. Koyama, A., Y. Niwa, H. Shigematsu, M. Taniguchi, and T. Tada. 1978. Studies on passive serum sickness. II. Factors determining the localization of antigen-antibody complexes in the murine renal glomerulus. Lab. Invest. 38: 253-262.

51. Stilmant, M. M., W. G. Couser, and R. S. Cotran. 1975 Experimental glomerulonephritis in the mouse associated with mesangial deposition of autologous ferritin immune complexes. Lab. Invest. 34: 746-756.

52. Rennke, H. G., R. S. Cotran, and M. A. Venkatachalam. 1975. Role of molecular charge in glomerular permeability: Tracer studies with cationized ferritin. J. Cell Biol. 67: 638-646.

53. Caulfield, J. P., and M. G. Farquhar. Distribution of anionic sites in glomerular basement membranes. Their possible role in filtration and attachment. Proc. Natl. Acad. Sci. U. S. A. 73: 1646-1650.

54. Sieler, M. W., M. A. Venkatachalam, and R. S. Cotran. 1975. Glomerular epithelium: Structural alterations induced by polycations. Science (Wash. D. C.). 189: 383-390. 
55. Seiler, M. W., H. G. Rennke, M. A. Venkatachalam, and R. S. Cotran. 1975. Pathogenesis of polycation-induced alterations ("fusion") of glomerular epithelium. Lab. Invest. 36: 48-61.

56. Ryan, G. B., S. J. Hein, and M. J. Karnovsky. 1976. Glomerular permeability to proteins. Effects of hemodynamic factors on the distribution of endogenous immunoglobulin $\mathbf{G}$ and exogenous catalase in the rat glomerulus. Lab. Invest. 34: 415-427.

57. Schneeberger, E. E., P. D. Leber, M. J. Karnovsky, and R. T. McCluskey. 1974. Altered functional properties of the renal glomerulus in autologous immune complex nephritis: An ultrastructural tracer study.J.Exp. Med. 139: 1283-1302.

58. Shibata, S., H. Sakaguchi, T. Nagasawa, and T. Naruse. 1972. Nephritogenic glycoprotein. II. Experimental production of membrane glomerulonephritis in rats by a single injection of homologous renal glycopeptide. Lab. Invest. 27: 457-465.

59. Couser, W. G., D. R. Steinmuller, D. J. Salant, M. M. Stilmant, and L. M. Lowenstein. 1978. Mechanisms of protein excretion in the isolated perfused rat kidney (IPK). Clin. Res. 26: 461. (Abstr.)

60. Nizet, A. 1975. The isolated perfused kidney: Possibilities, limitations and results. Kidney Int. 7: 1-11.

61. Kniker, W. T., and C. G. Cochrane. 1968. The localization of circulating immune complexes in experimental serum sickness: The role of vasoactive amines and hydrodynamic forces. J. Exp. Med. 127: 119-135.

62. Wilson, C. B., and F. J. Dixon. 1976. The renal response to immunological injury. In The Kidney. B. M. Brenner and F. C. Rector, Jr., editors. W. G. Saunders Company, Philadelphia. 1st edition. 838-940. 